\title{
Examination of the Chance Constrained Optimal WT Penetration Level in Distorted Distribution Network with Wind Speed and Load Uncertainties
}

\author{
Ibrahim Cagri Barutcu \\ Department of Electricity and Energy \\ Çölemerik V.H.S. \\ Hakkari University \\ Hakkari, Turkey \\ ibrahimcagribarutcu@hakkari.edu.tr
}

\begin{abstract}
Harmonic penetration can be problematic by the growing interconnection of Wind Turbines (WTs) in distribution networks. Since the active power outputs of WTs and loads in the distribution system have uncertainties, the optimal WT penetration level problem can be considered to have a stochastic nature. In this study, this problem is taken into account in the stochastic optimization method with the consideration of uncertainties in wind speed and distribution network load profile. Chance constraint programming is taken into account in the determination of optimal WT penetration levels by applying the Genetic Algorithm (GA) along with Monte Carlo Simulation (MCS). The harmonic power flow analysis based on the decoupled harmonic load flow approach is employed in the distorted distribution network. Chance constraints are considered for the harmonic issues such as the Total Harmonic Distortion of Voltage (VTHD), Individual Harmonic Distortion of Voltage (VIHDh), and Root Mean Square of Voltage (VRMS).
\end{abstract}

Keywords-chance constraint programming; wind turbine; distribution network; stochastic optimization

\section{INTRODUCTION}

Wind Turbines (WTs) are gaining importance with the increase in power demand in distribution systems [1]. These DG systems, which are integrated to distribution systems with power electronics converters, have a wide range of applications. Although WTs meet the power demand in the distribution networks, they can also have negative effects. The fact that the penetration levels of WTs have increased significantly causes problems in terms of power quality issues related to harmonic distortion in distribution systems [2-4]. These types of problems result from the power electronics converter interfaces that provide the interconnection between the WTs and the distribution network. Currents and voltages are increasingly diverging from their sinusoidal shapes since power electronics devices have been widely used in distribution networks. This situation negatively affects the quality of the power transmitted to the consumers. As a result of high installation of WTs in distribution networks with nonlinear loads, high levels of harmonics cause negative results in terms of efficiency and reliability of distribution systems. In this manner, it is useful to optimize the penetration level of WTs in the network by taking the harmonic parameters into consideration. Therefore, the examination of the influence of WTs on the harmonic issues of power systems is a vital problem [5]. On the other hand, the investigations on the harmonic impact of WTs on the distribution systems require information about the harmonic characteristics of their output currents. In this manner, the increasing penetration of WTs plays an important role in the evaluation of the harmonic influences of these systems. Wind speed variations give rise to changes in the operating points of WTs. This in turn influences the harmonic distortions caused by the output current of the wind system. In general, the problem concerning the impacts of wind speed changes on harmonic power quality of the utility grid is considered in the planning of optimal placement and capacity of WTs on distorted distribution networks.

Some studies consider the effects of wind speed on the behavior of harmonic power quality of the power system [610]. In [6], the harmonic influences of wind system on the grid power quality have been examined, and it has been shown that variable speed conditions cause greater Total Harmonic Distortion (THD) current when compared to constant speed operation. While installing the WTs at power grids, the assessment of harmonic power quality of the power network is a very important issue due to wind speed variations. In [7, 8], the output current of the Doubly Fed Induction Generator (DFIG) type of WT has been examined in case of instantaneous variations in wind speed. It has been determined that the harmonic frequency currents have stochastic behavior due to the uncertainty in wind speed conditions. It is important to note that the dependency of harmonic problems on wind turbine operation conditions such as wind speed variations is a vital problem in the literature. In [9], the impact of these conditions on the harmonic output currents of the WT has been investigated. It has been concluded that these harmonic currents can be represented by probability distributions because of the random characteristics based on the changes in the wind speed. The harmonic current components of a wind farm have been analyzed by taking into account the relations between the 
harmonic injection currents and the output powers of the WTs in [10].

Optimal planning of inverter-based DGs considering harmonic penetration is generally performed using deterministic optimization procedures [11]. On the other hand, the random nature of problems may not be taken into account in the deterministic approaches, which present conservative behavior in terms of optimization solutions. However, the random characteristics of environmental conditions and distribution system load profile make the optimization problems to have probabilistic nature. The optimization outcomes can be represented by the probability distributions within the determined region by utilizing the probabilistic approaches. In this manner, Monte Carlo Simulation (MCS) is a well suited method for handling the probabilistic and harmonic related problems [12-14]. In [12], an optimization problem has been investigated by the MCS method with the consideration of confidence intervals for THD current limits in distribution system with background harmonics. Chance constrained programming plays important role in performing the planning of distorted distribution systems. In [13], MCSbased Genetic Algorithm (GA) optimization has been applied to determine the optimal planning of passive harmonic filters considering the confidence intervals for harmonic constraints. In [14], a method incorporating GA and MCS has been proposed for optimal planning of passive harmonic filters taking into account chance constraint for VTHD in a distribution network, consisting of PV systems and nonlinear loads.

However, the optimization framework for planning the WT penetration level by simultaneously considering the chance constraints for the harmonic power quality indices, which are VTHD, VIHDh and VRMS has not been taken into account in these studies. In the present paper, these literature gaps have been handled in detail. In the current paper, the optimal WT penetration level problem is taken into account in the stochastic optimization method with the consideration of wind speed and load uncertainties in the distribution system. Chance constraint programming is considered in the determination of optimal WT penetration levels by performing GA along with MCS. The harmonic power flow analysis based on decoupled harmonic load flow method is employed. The chance constraints are considered for VTHD, VIHDh, and VRMS.

\section{HARMONIC POWER FLOW}

As the penetration levels of WTs increase in distorted distribution networks with nonlinear loads, harmonics also increase. WT harmonics negatively affect devices in distribution networks with higher background harmonics. As the interconnection of WTs in the distribution system rises, the total harmonic distortions in the voltage and current cause the lifetime of devices to decrease. In this context, the harmonic issues must be considered when integrating WTs into distribution networks, which are distorted. Harmonic distortions in electricity distribution networks increase due to the inverter interfaces in the structures of WTs resulting from the installation of these sources into the distribution grids. In addition, nonlinear loads, which are widely used in distribution systems, have an important role in these harmonic distortions.
As a result of the constantly increasing energy demands, power quality is a major issue. The nonlinear loads and the variability of WT active power capacities, which depend on wind speed, may affect the power quality in distribution systems. In this context, harmonic power flow analysis studies play an important role in terms of analysing and designing distribution systems considering harmonic distortions. In this manner, THD becomes higher when the WTs meet all the power demands in the distribution network, with nonlinear loads also connected. Therefore, harmonic analysis study is inevitable for the examination of power quality issues.

In this paper, the harmonic analysis based on decoupled approach is utilized [15]. The harmonic power flow can be solved by taking into account the following equation for each harmonic order of interest:

$$
I_{B u s}^{h}=Y_{B u s}^{h} V_{B u s}^{h}
$$

where $Y_{B u s}^{h}$ represents the bus admittance matrix, $V_{B u s}^{h}$ shows the bus voltage vector, and $I_{B u s}^{h}$ denotes the total harmonic current injection vector of wind turbines and nonlinear loads.

\section{OPTIMIZATION FRAMEWORK}

In this article, GA [16] is carried out to maximize the optimal WT penetration level based on the constraints. This problem can be considered to have stochastic nature since the active power outputs of WTs and loads in the distribution network have uncertainties. Thus, the chance constraints are taken into account for the VTHD, VIHDh, and VRMS limits. The determination of the optimal WT penetration levels based on chance constraint programming is handled by performing GA along with MCS. The objective function can be presented as:

$$
P L(\%)=\frac{\sum_{k=1}^{N c} P_{W T, k}}{\text { Total }_{M V A}} x 100
$$

where $N c$ is the amount of WT candidate buses, $P_{W T, k}$ is the wind turbine capacity at bus $k$, Total $_{M V A}$ shows the total system MVA of the distribution system, and PL $(\%)$ denotes the total penetration level of WTs. The chance constraints for the power quality indices are given by:

$$
\begin{gathered}
\operatorname{Pr}\left\{V T H D^{k}(\%) \leq V T H D^{k, \max }(\%)\right\} \geq \alpha_{V T H D} \\
\operatorname{Pr}\left\{V I H D h^{k}(\%) \leq V I H D h^{k, \max }(\%)\right\} \geq \alpha_{V I H D h} \\
\operatorname{Pr}\left\{V R M S^{k, \min } \leq V R M S^{k} \leq V R M S^{k, \max }\right\} \geq \alpha_{V R M S}
\end{gathered}
$$

where:

$$
\operatorname{VTHD}^{k}(\%)=\frac{\sqrt{\sum_{h}^{H}\left|V^{h, k}\right|^{2}}}{\left|V^{1, k}\right|} \times 100
$$




$$
\begin{gathered}
\operatorname{VIHDh}^{k}(\%)=\frac{\left|V^{h, k}\right|}{\left|V^{1, k}\right|} x 100 \\
V R M S^{k}=\sqrt{\left|V^{1, k}\right|^{2}+\sum_{h}^{H}\left|V^{h, k}\right|^{2}}
\end{gathered}
$$

$V^{1, k}, V^{h, k}, V T H D^{k}, V I H D h^{k}$, and $V R M S^{k}$, denote fundamental frequency voltage, harmonic frequency voltage, total harmonic distortion of voltage, individual harmonic distortion of voltage, and root mean square of voltage at bus $k$, respectively. $h$ and $H$ are the harmonic order of interest and maximum harmonic order respectively. $\alpha_{V T H D}, \alpha_{V I H D h}$, and $\alpha_{V R M S}$ show the confidence levels (CL) for $V T H D^{k}, V I H D h^{k}$, and $V R M S^{k}$ respectively.

The overall objective function has been determined considering the chance constraint violations in harmonic parameters with the utilization of penalty function approach [17]:

$$
\begin{aligned}
& f_{\text {overall }}=\frac{\sum_{k=1}^{N c} P_{W T, k}}{\operatorname{Total}_{M V A}} x 100 \\
& +p m_{\text {VTHD }} \sum_{k=1}^{T B}\left|\varphi_{V T H D}^{k}-\left(1-\alpha_{V T H D}\right)\right| \\
& +p m_{\text {VIHDh }} \sum_{k=1}^{T B} \sum_{h}^{H}\left|\varphi_{V I H D h}^{k}-\left(1-\alpha_{V I H D h}\right)\right| \\
& +p m_{\text {VRMS }} \sum_{k=1}^{T B}\left|\varphi_{V R M S}^{k}-\left(1-\alpha_{\text {VRMS }}\right)\right|
\end{aligned}
$$

where:

$$
\begin{gathered}
\varphi_{V T H D}^{k}=1-\operatorname{Pr}\left\{V \operatorname{VHH}^{k}(\%) \leq V T H D^{k, \max }(\%)\right\} \\
\varphi_{V I H D h}^{k}=1-\operatorname{Pr}\left\{V I H D h^{k}(\%) \leq V I H D h^{k, \max }(\%)\right\} \\
\varphi_{V R M S}^{k}=1-\operatorname{Pr}\left\{V R M S^{k, \min } \leq V R M S^{k} \leq V R M S^{k, \max }\right\}
\end{gathered}
$$

where $p m_{V T H D}, p m_{V I H D h}$, and $p m_{V R M S}$ represent the penalty multipliers in case of the violations in chance constraints corresponding to VTHD, VIHDh, and VRMS respectively and $\varphi_{V T H D}^{k}, \varphi_{V I H D h}^{k}$, and $\varphi_{V R M S}^{k}$ are the probabilities of limit violations in VTHD, VIHDh, and VRMS respectively. TB is the total amount of nodes in the distribution network. As illustrated in (9), the WT penetration level is maximized provided that the probabilities of violations are within the corresponding limits. When the probabilities of violations exceed their limits, the penalty factors are utilized. Therefore, the optimization algorithm explores a more suitable solution, when the overall objective function becomes larger due to the violations in chance constraints. In this paper, GA is utilized to handle the stochastic optimization problem illustrated in (9). The solution representing the optimal active output powers of WTs at each candidate node of distribution network is presented as follows:

$$
X=\left(P_{W T, 1}, \ldots, P_{W T, k}, \ldots, P_{W T, N c}\right)
$$

The lower and upper limits of wind turbine capacities are presented as in:

$$
P_{W T, \text { min }} \leq P_{W T, k} \leq P_{W T, \text { max }}
$$

The main steps of the overall methodology, which is performed by applying GA in conjunction with MCS, are:

1. Input the parameters that belong to the distribution system.

2. Input the harmonic spectrums of the WT system and nonlinear loads.

3. Generate 1000 different wind speed and load states from the corresponding probability distributions.

4. Generate the initial population representing the candidate solutions randomly.

5. Start the MCS for the $t^{\text {th }}$ state $(t=1,2, \ldots, 1000)$, otherwise, if $\mathrm{t}$ is greater than 1000 , go to step 10 .

6. Determine the active power outputs of WT systems and get the load data corresponding to the $t^{\text {th }}$ state.

7. Implement the decoupled harmonic power flow method for the $t^{\text {th }}$ state.

8. Evaluate the VTHD, VIHDh, and VRMS at each bus of the distribution system for the $t^{\text {th }}$ state.

9. Go to step 5 .

10. Determine the probability density functions of distributions of VTHD, VIHDh, and VRMS at all buses of the distribution system by considering all states.

11. Calculate the probabilities of limit violations in VTHD, VIHDh, and VRMS by taking into account the probability density functions of these parameters. In this step, the probabilities of violations are determined by integrating the probability density functions over the intervals of limit violations for VTHD, VIHDh, and VRMS at every bus of the distribution system [18]. As an example, this process is illustrated in Figure 1 for the harmonic power quality parameters at bus $k$ of the distribution network. The total and individual harmonic distortions of voltage have been limited by $5 \%$ and $3 \%$ respectively [19], according to the IEEE 519 Standards. The root mean square value of voltage has the lower and upper bounds of $0.9 \mathrm{pu}$ and $1.1 \mathrm{pu}$ respectively. The shaded areas under the probability density functions represent the probability of limit violations, except for the shaded areas which illustrate the confidence level for the harmonic parameters.

12. Check the chance constraints whether they are within their limits or not. The chance constraints are satisfied when the areas under the probability density functions of VTHD, VIHDh, and VRMS over the corresponding intervals of 
limit violations are less than $1-\alpha_{V T H D}, 1-\alpha_{V I H D h}$, and $1-\alpha_{V R M S}$ respectively.

13. Calculate the objective function for each chromosome considering the chance constraints related to the harmonic power quality indices.

14. Evaluate the objective function by taking into account the penalty function method, if the chance constraints are not met.

15. Print the optimal active power outputs of WTs if the optimization criteria are satisfied, otherwise go to step 4 .

16. Test the optimization results considering the chance constraint violations for harmonic power quality parameters.

(a)

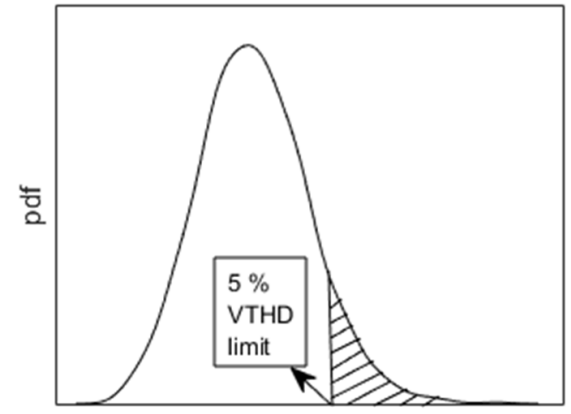

VTHD at Bus k (\%)

(b)

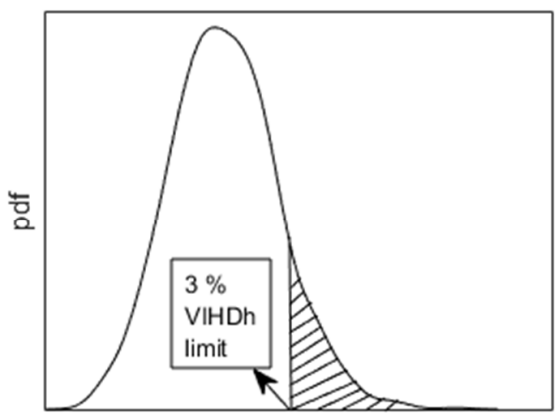

VIHDh at Bus $\mathrm{k}(\%)$

(c)

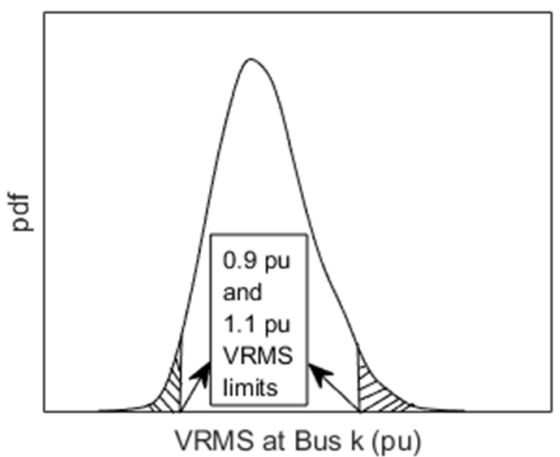

Fig. 1. The probability density functions of (a) VTHD, (b) VIHDh, (c) VRMS at Bus k.
The chance constrained optimization method is utilized for the probabilistic optimization problems, the constraints of which have random characteristics. Determination of the optimal solution means that the stochastic constraints are within their limits for the defined confidence interval. In this context, MCS is a well suited method for dealing with the probabilistic problems. The flowchart of GA optimization methodology along with MCS is demonstrated in Figure 2. The flowchart of the approach for testing the optimization results by taking into account the chance constraints is given in Figure 3.

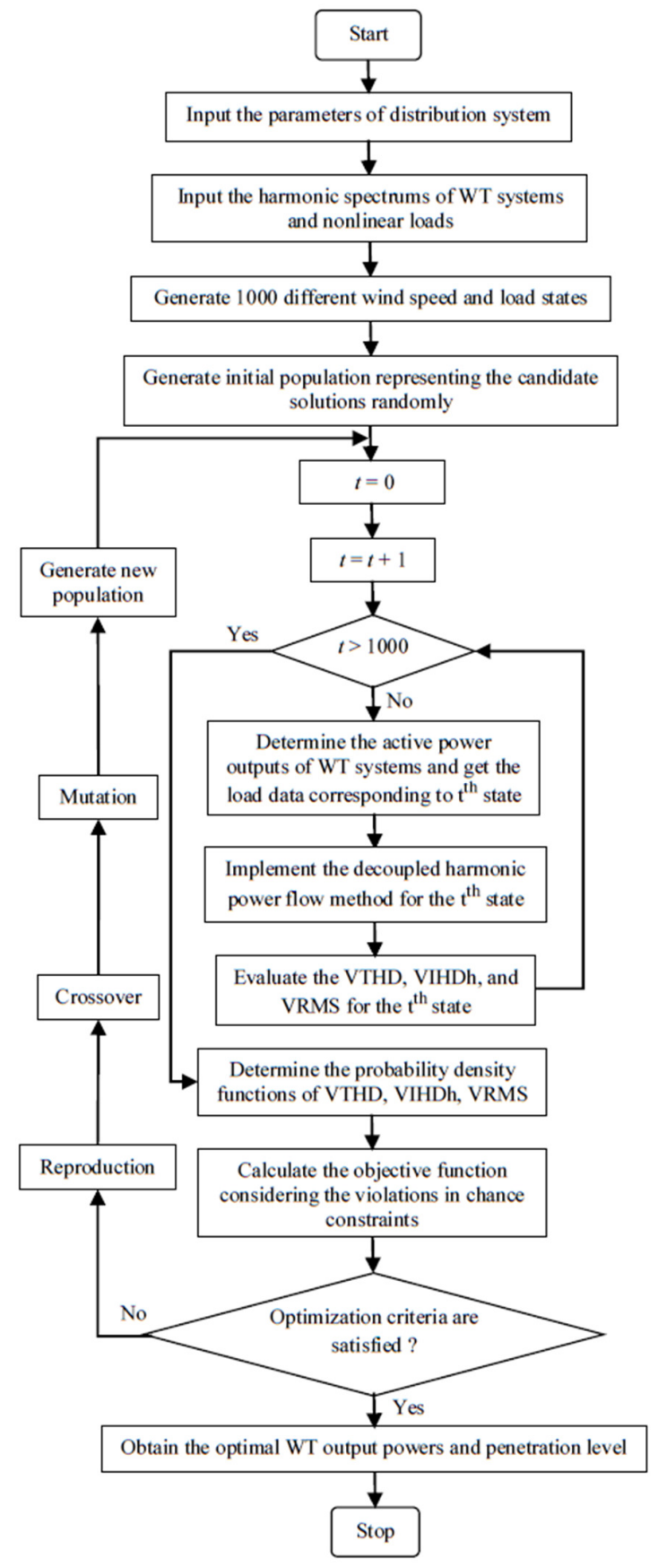

Fig. 2. The flow chart of the proposed method. 


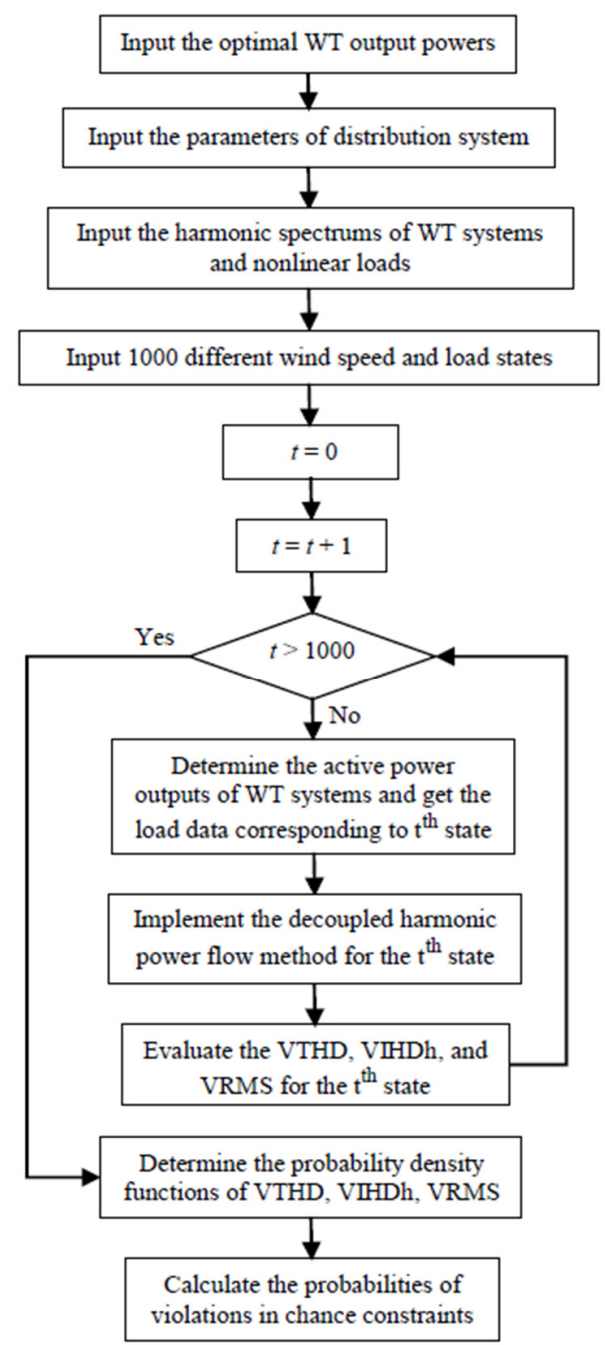

Fig. 3. The flowchart of the approach for testing the optimization results.

In this paper, the 1000 different wind speed and load states have been firstly generated from their corresponding probability distributions. Then, the GA optimization process has been initiated by randomly generating initial populations representing the candidate solutions. The MCS approach, which has been embedded in the optimization algorithm, has been applied for all wind speed and load states. The decoupled harmonic power flow method has been implemented to evaluate VTHD, VIHDh, and VRMS at each bus of the distribution system by considering the active power outputs of WT systems and load data for all states in the MCS algorithm. After the MCS process has been completed, the probability density functions of VTHD, VIHDh, and VRMS have been determined. After that, the probabilities of limit violations in these harmonic parameters have been calculated taking into account the probability density functions. The objective function has been evaluated with the consideration of limit violations in chance constraints. If the chance constraints have not been met, the objective function has been calculated by considering the penalty function method. GA optimization approach has been iteratively carried out by considering reproduction, mutation, crossover, and the new population generation stages until the optimization criteria were satisfied. After the optimization process presented in Figure 2 has been completed, the optimization results have been tested to determine the probabilities of violations in chance constraints by using the method illustrated in Figure 3.

\section{MODELING OF UNCERTAINTIES}

The uncertainties of load demand and wind speed are gaining importance in the stochastic power flow analysis of distribution networks with the growing interconnection of wind turbines to these power systems. The probabilistic approaches, in which these uncertainties are taken into account, are inevitable for the evaluation of the stochastic behavior of distribution systems, to which the WTs are interfaced. In that manner, it is very important for stochastic approaches that the random variables in the problem have some probability distributions [12]. Since the consumptions and renewable power productions in distribution systems have probabilistic framework, these are included in the stochastically modeled approaches. The outcomes, which are determined by the probabilistic approach, also show stochastic behavior and follow a probabilistic distribution. The output random variables of stochastic process performed on the problem have uncertainties and probabilistic features as the input ones have these properties [20]. The distribution functions are generally utilized to express the uncertainties of load demand and wind speed in probabilistic methods.

In this paper, the wind speed is considered to have a Rayleigh Distribution [21]. The probability density function (pdf) of wind speed is given as in the following:

$$
f\left(v_{W T, k}\right)=\left(\frac{2 v_{W T, k}}{c^{2}}\right) \exp \left(-\left(\frac{v_{W T, k}}{c}\right)^{2}\right)
$$

where $c$ represents scale index and $v_{W T, k}$ shows the wind speed on the wind turbine at bus $k$ of the distribution network. The wind turbine capacity depends on wind speed, and can be evaluated as [21]:

$$
P_{W T, k}=\left\{\begin{array}{cc}
0 & 0 \leq v_{W T, k} \leq v_{c, i} \\
\left(\frac{v_{c, i}-v_{W T, k}}{v_{c, i}-v_{W T, N}}\right) P_{W T, N} & v_{c, i} \leq v_{W T, k} \leq v_{W T, N} \\
P_{W T, N} & v_{W T, N} \leq v_{W T, k} \leq v_{c, o} \\
0 & v_{c, o} \leq v_{W T, k}
\end{array}\right.
$$

where $v_{W T, N}$ represents the nominal speed of WT, $v_{c, o}$ shows the cut-out speed of WT, $v_{c, i}$ illustrates the cut-in speed of WT, and $P_{W T, N}$ is the nominal active power output of WT.

In this study, the load uncertainty of the distribution network has been modelled by normal distribution [12]. The normal distribution pdf is given as follows: 


$$
f\left(P_{D, k}\right)=\left(\frac{1}{\sqrt{2 \pi} \sigma}\right) \exp \left(-\frac{\left(P_{D, k}-P_{D, k, \text { mean }}\right)^{2}}{2 \sigma^{2}}\right)
$$

where $P_{D, k}$ illustrates the active power load at bus $k$ of distribution system, $P_{D, k, \text { mean }}$ is the load profile mean value at bus $k$, and $\sigma$ is the standard deviation.

\section{SimUlation RESUlts}

In this study, GA parameters are selected as: $10^{-6}$ for the tolerance of fitness function, 200 for the maximum number of generation, 30 for population size, and 0.8 for the crossover fraction. The study simulations have been implemented on a PC with $2.80 \mathrm{GHz}$ CPU. In this study, the optimal penetration levels of WTs have been determined based on the uncertainties of load powers on the distribution network and wind speed on WTs. The chance constraints for harmonic issues have been considered in the stochastic planning of active output powers of WTs. The WT penetration levels have been maximized by ensuring chance constraint limits and considering a probabilistic framework of load powers and wind speed. The WTs have the installed capacity of $500 \mathrm{~kW}$ at each candidate bus of the distribution system. A 33 bus distribution network [22] has been used to perform the simulations. This system, which has the total capacity of 6MVA, is given in Figure 4. The chosen WT candidate buses are 5, 11, 16, 17, 19, 22, 24, $25,26,28,30$ and 32 .

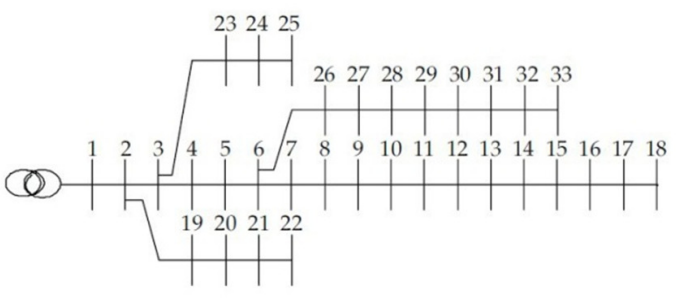

Fig. $4 . \quad 33$ bus radial test system.

Different states have been generated for the load profile of the distribution system. The active powers of original load in the grid are regarded as the mean values. The generated load cases have $10 \%$ standard deviation with respect to the mean values. The total distribution system active load profile is given in Figure 5. The proposed methodology considers the examination of harmonic power quality indices on WTs integrated distribution system based on the uncertainties in the probabilistic optimization framework. Nominal, cut - out, and cut - in speeds of WTs have been regarded as $16 \mathrm{~m} / \mathrm{s}, 25 \mathrm{~m} / \mathrm{s}$, and $4 \mathrm{~m} / \mathrm{s}$ respectively [23]. The wind speed states have been generated based on the Rayleigh Distribution in the proposed method. The uncertainties in wind speed conditions, which in turn affect the WT power generation, give rise to the probabilistic generation in WT systems. As a result of wind speed uncertainties in WT systems and load uncertainties in distribution systems, the probabilistic approaches are applied. By the aid of the probabilistic model, the different cases of random variables can be considered in the active distribution network, which is examined. The probabilistic approach requires handling the input random variables, which are represented by distribution functions, by performing deterministic approaches for different states. By implementing the probabilistic method with the inclusion of input random variables, the output variables, which also follow a distribution function, can be determined. The wind speed states, which are generated from the corresponding probability distribution, are illustrated in Figure 6.

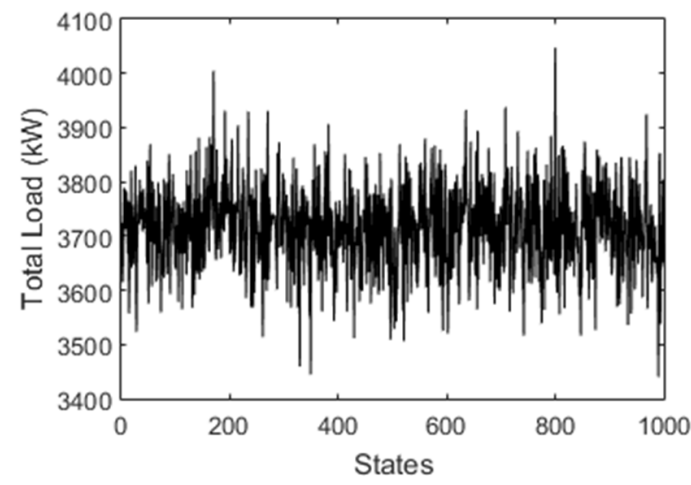

Fig. 5. The total load states of the distribution system.

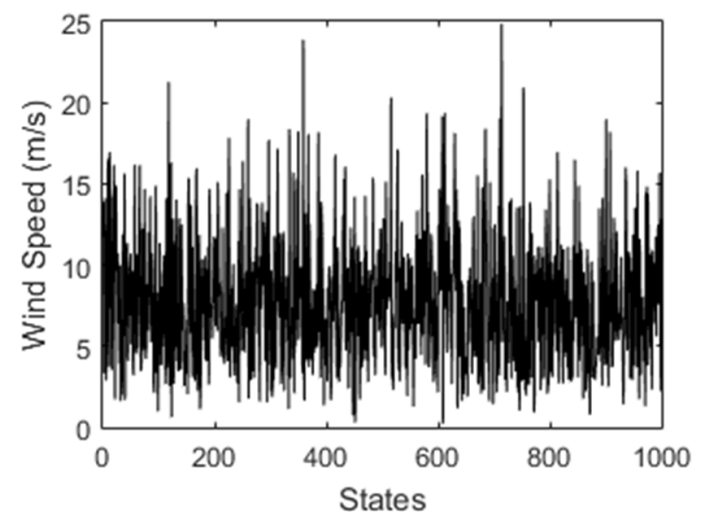

Fig. 6. The wind speed states.

The installation nodes and the capacities of nonlinear loads are presented in Table I. The harmonic current components of WTs and nonlinear loads are demonstrated in Table II $[11,15]$.

TABLE I. NONLINEAR LOAD POWERS IN THE DISTRIBUTION SYSTEM

\begin{tabular}{|c|c|c|}
\hline Buses & Active Power (kW) & Reactive Power (kVar) \\
\hline 2 & 45 & 27 \\
\hline 6 & 27 & 9 \\
\hline 14 & 54 & 36 \\
\hline 19 & 40.5 & 18 \\
\hline 23 & 40.5 & 22.5 \\
\hline 27 & 27 & 11.25 \\
\hline 33 & 27 & 18 \\
\hline
\end{tabular}

The optimal active power outputs of WTs are shown for different confidence levels in Table III by performing GA with MCS in chance constraint optimization framework. The optimal values of WT penetration levels have been determined 
as $44.79 \%, 40.86 \%$, and $36.28 \%$ for $0.7,0.8$, and 0.9 confidence levels respectively. The GA success rates [24] determined for $0.7,0.8$, and 0.9 confidence levels are depicted in Figure 7. The GA optimization approach, where MCS is embedded, has converged to the optimal solutions in $26556.55 \mathrm{~s}$ at 89 iterations for 0.7 confidence level, in $25189.85 \mathrm{~s}$ at 94 iterations for 0.8 confidence level, and in $38654.93 \mathrm{~s}$ at 143 iterations for 0.9 confidence level respectively.

TABLE II. HARMONIC CURRENT COMPONENTS

\begin{tabular}{|c|c|c|}
\hline \multirow{2}{*}{ Harmonic Order } & \multicolumn{2}{|c|}{ Magnitudes (\%) } \\
\cline { 2 - 3 } & Wind Turbine & Nonlinear Load \\
\hline 1 & 100 & 100 \\
\hline 5 & 4.00 & 23.52 \\
\hline 7 & 4.00 & 6.08 \\
\hline 11 & 2.00 & 4.57 \\
\hline 13 & 2.00 & 4.20 \\
\hline 17 & 1.50 & 1.80 \\
\hline 19 & 1.50 & 1.37 \\
\hline 23 & 0.60 & 0.75 \\
\hline 25 & 0.60 & 0.56 \\
\hline 29 & 0.60 & 0.49 \\
\hline 31 & 0.60 & 0.54 \\
\hline 35 & 0.30 & - \\
\hline 37 & 0.30 & - \\
\hline 41 & 0.30 & - \\
\hline 43 & 0.30 & - \\
\hline 47 & 0.30 & - \\
\hline 49 & 0.30 & - \\
\hline
\end{tabular}

TABLE III. OPTIMAL WT OUTPUT POWERS FOR DIFFERENT CONFIDENCE LEVELS

\begin{tabular}{|c|c|c|c|}
\hline \multirow{2}{*}{ Buses } & \multicolumn{3}{|c|}{$\boldsymbol{P}_{\boldsymbol{W T}, \boldsymbol{k}}(\mathbf{k W})$} \\
\cline { 2 - 4 } & For 0.7 CL & For 0.8 CL & For 0.9 CL \\
\hline 5 & 379.33 & 396.67 & 78.39 \\
\hline 11 & 59.53 & 59.88 & 261.31 \\
\hline 16 & 274.82 & 374.02 & 371.44 \\
\hline 17 & 128.11 & 4.05 & 83.66 \\
\hline 19 & 327.05 & 83.26 & 178.25 \\
\hline 22 & 137.18 & 208.05 & 55.33 \\
\hline 24 & 382.04 & 243.40 & 217.03 \\
\hline 25 & 133.48 & 138.96 & 18.91 \\
\hline 26 & 197.78 & 343.86 & 235.66 \\
\hline 28 & 308.93 & 288.38 & 250.29 \\
\hline 30 & 225.41 & 211.59 & 215.29 \\
\hline 32 & 134.13 & 99.58 & 211.32 \\
\hline
\end{tabular}

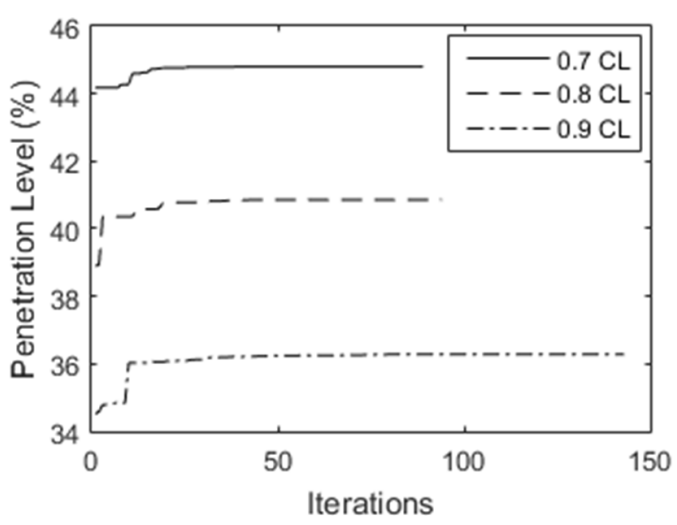

Fig. 7. The iteratively depicted optimal WT penetration levels.
As presented in Table III, the optimal WT penetration levels decrease with the increase of confidence levels. The main reason is that the optimization process is more conservative in higher confidence levels. Since the optimal penetration levels of WTs in lower confidence levels are less than those at the higher levels by the chance constraints, the optimal WT penetration levels are increasing by the decrease of confidence levels. The upper limits and optimal values of WT active power outputs for different confidence levels have been interconnected to the distribution network to test violations in chance constraints related with harmonic power quality parameters. The violations in chance constraints for these parameters are presented in Table IV.

As seen in Table IV, chance constraint violations of VTHD, VIHD5, VIHD7, and VRMS are observed when the nominal capacities of WT output powers are connected to the distribution system. The optimal active power outputs by the proposed methodology cause considerable minimization in the violations. These optimal output powers of WTs ensure that the violations in the chance constraints of harmonic related issues comply with their corresponding limits for each confidence level. The chance constraint violations of VIHD7 are not observed for the optimal solutions of WT output powers for all confidence levels. The violations in chance constraints for power quality parameters are satisfied with the corresponding confidence levels, in which the probabilistic optimization procedure has been applied. According to [25], meaningful results can be determined by using 1000 states in the MCS process. Low accuracy can be increased by increasing the number of states. This in turn does not require the utilization of a greater number of states. In this study, the wind speed states in Figure 6 and the load states in Figure 5, which are considered in the GA optimization process, are also taken into account in the testing of the optimization results. The chance constraints are satisfied by the GA optimization results for these data sets. It can be concluded that the load and wind speed data sets, which are taken into account in the optimization algorithm, illustrate the chance constraint handling ability of the optimization process when they are used as test sets.

By the increase of WT integration into the distribution systems, harmonic distortions cause negative effects in these networks. The connection of these renewable energy resources to the grids with nonlinear loads leads the distribution system planners to research on ensuring harmonic power quality. In this context, determining the optimal WT penetration levels in active distribution networks, where high harmonic currents and voltages are observed, is a major issue. Optimal penetration levels of WTs obtained based on the harmonic limits specified in the IEEE 519 standards become important for the continuity of harmonic power quality in the distribution systems. Maintaining power quality is inevitable for the efficiency and reliability of power transferred to the end users in the distribution network. In this manner, the occurrence of harmonic problems in the distribution systems requires that each phase of planning for the optimal penetration levels of wind turbines should be made correctly. 
TABLE IV. VIOLATION PROBABILITIES

\begin{tabular}{|c|c|c|c|c|c|c|c|c|c|c|c|c|c|}
\hline \multirow{3}{*}{ Buses } & \multicolumn{13}{|c|}{ Violation probabilities in harmonic power quality param eters } \\
\hline & \multicolumn{4}{|c|}{ For $P_{W T, \max }$} & \multicolumn{3}{|c|}{$P_{W T, k}$ for $0.7 \mathrm{CL}$} & \multicolumn{3}{|c|}{$P_{W T, k}$ for $0.8 \mathrm{CL}$} & \multicolumn{3}{|c|}{$P_{W T, k}$ for $0.9 \mathrm{CL}$} \\
\hline & VTHD & VIHD5 & VIHD7 & VRMS & VTHD & VIHD5 & VRMS & VTHD & VIHD5 & VRMS & VTHD & VIHD5 & VRMS \\
\hline 1 & 0.5073 & 0.8984 & 0.1610 & - & 0.0007 & 0.0748 & - & - & 0.0379 & - & - & 0.0111 & - \\
\hline 2 & 0.5083 & 0.9131 & 0.1617 & - & 0.0007 & 0.0750 & - & - & 0.0380 & - & - & 0.0111 & - \\
\hline 3 & 0.5437 & 0.9146 & 0.1835 & - & 0.0008 & 0.0859 & - & - & 0.0447 & - & - & 0.0145 & - \\
\hline 4 & 0.5469 & 0.9155 & 0.1855 & - & 0.0009 & 0.0978 & - & - & 0.0522 & - & - & 0.0168 & - \\
\hline 5 & 0.5503 & 0.9288 & 0.1877 & - & 0.0010 & 0.0984 & - & - & 0.0525 & - & - & 0.0169 & - \\
\hline 6 & 0.5875 & 0.9410 & 0.1916 & - & 0.0011 & 0.1277 & - & 0.0003 & 0.0706 & - & - & 0.0235 & - \\
\hline 7 & 0.5890 & 0.9408 & 0.2137 & - & 0.0012 & 0.1450 & - & 0.0003 & 0.0812 & - & - & 0.0278 & - \\
\hline 8 & 0.5964 & 0.9508 & 0.2193 & - & 0.0014 & 0.1659 & - & 0.0008 & 0.1066 & - & - & 0.0391 & - \\
\hline 9 & 0.6315 & 0.9513 & 0.2228 & - & 0.0016 & 0.1886 & - & 0.0017 & 0.1216 & - & - & 0.0543 & - \\
\hline 10 & 0.6368 & 0.9595 & 0.2504 & - & 0.0019 & 0.2389 & - & 0.0022 & 0.1383 & - & - & 0.0636 & - \\
\hline 11 & 0.6377 & 0.9596 & 0.2511 & - & 0.0019 & 0.2392 & - & 0.0022 & 0.1384 & - & - & 0.0637 & - \\
\hline 12 & 0.6388 & 0.9597 & 0.2520 & - & 0.0019 & 0.2396 & - & 0.0022 & 0.1564 & - & - & 0.0639 & - \\
\hline 13 & 0.6736 & 0.9667 & 0.2568 & - & 0.0026 & 0.2976 & - & 0.0031 & 0.1990 & 0.0001 & - & 0.0867 & 0.0001 \\
\hline 14 & 0.6771 & 0.9669 & 0.2593 & 0.0002 & 0.0026 & 0.2990 & 0.0005 & 0.0034 & 0.1998 & 0.0011 & 0.0001 & 0.1000 & 0.0009 \\
\hline 15 & 0.6791 & 0.9669 & 0.2611 & 0.0004 & 0.0026 & 0.2992 & 0.0013 & 0.0034 & 0.1999 & 0.0026 & 0.0001 & 0.0998 & 0.0017 \\
\hline 16 & 0.6814 & 0.9668 & 0.2634 & 0.0008 & 0.0026 & 0.2994 & 0.0022 & 0.0034 & 0.2000 & 0.0083 & 0.0001 & 0.0996 & 0.0033 \\
\hline 17 & 0.6849 & 0.9664 & 0.2672 & 0.0015 & 0.0026 & 0.3000 & 0.0068 & 0.0034 & 0.2000 & 0.0215 & 0.0001 & 0.0993 & 0.0089 \\
\hline 18 & 0.6849 & 0.9664 & 0.2672 & 0.0015 & 0.0026 & 0.3000 & 0.0093 & 0.0034 & 0.2000 & 0.0288 & 0.0001 & 0.0993 & 0.0123 \\
\hline 19 & 0.5086 & 0.9132 & 0.1619 & - & 0.0007 & 0.0750 & - & - & 0.0380 & - & - & 0.0126 & - \\
\hline 20 & 0.5099 & 0.9137 & 0.1629 & - & 0.0007 & 0.0747 & - & - & 0.0376 & - & - & 0.0126 & - \\
\hline 21 & 0.5104 & 0.9139 & 0.1632 & - & 0.0007 & 0.0745 & - & - & 0.0375 & - & - & 0.0125 & - \\
\hline 22 & 0.5115 & 0.9002 & 0.1638 & - & 0.0007 & 0.0742 & - & - & 0.0371 & - & - & 0.0125 & - \\
\hline 23 & 0.5445 & 0.9150 & 0.1840 & - & 0.0009 & 0.0862 & - & - & 0.0448 & - & - & 0.0145 & - \\
\hline 24 & 0.5461 & 0.9156 & 0.1850 & - & 0.0008 & 0.0862 & - & - & 0.0448 & - & - & 0.0145 & - \\
\hline 25 & 0.5166 & 0.9158 & 0.1856 & - & 0.0008 & 0.0862 & - & - & 0.0446 & - & - & 0.0145 & - \\
\hline 26 & 0.5881 & 0.9412 & 0.1921 & - & 0.0011 & 0.1279 & - & 0.0003 & 0.0812 & - & - & 0.0279 & - \\
\hline 27 & 0.5889 & 0.9414 & 0.2140 & - & 0.0011 & 0.1282 & - & 0.0003 & 0.0814 & - & - & 0.0279 & - \\
\hline 28 & 0.5906 & 0.9421 & 0.2157 & - & 0.0013 & 0.1463 & - & 0.0005 & 0.0819 & - & - & 0.0280 & - \\
\hline 29 & 0.5921 & 0.9425 & 0.2169 & - & 0.0013 & 0.1470 & - & 0.0005 & 0.0939 & - & - & 0.0332 & - \\
\hline 30 & 0.5930 & 0.9428 & 0.2176 & - & 0.0013 & 0.1473 & - & 0.0005 & 0.0940 & - & - & 0.0333 & - \\
\hline 31 & 0.5943 & 0.9430 & 0.2183 & - & 0.0014 & 0.1675 & - & 0.0008 & 0.1075 & - & - & 0.0394 & - \\
\hline 32 & 0.5949 & 0.9522 & 0.2186 & - & 0.0014 & 0.1679 & - & 0.0008 & 0.1077 & - & - & 0.0394 & - \\
\hline 33 & 0.5952 & 0.9523 & 0.2187 & - & 0.0014 & 0.1898 & - & 0.0008 & 0.1080 & - & - & 0.0465 & - \\
\hline
\end{tabular}

Harmonic studies carried out in distribution networks, are a major issue these networks face. Studies which find applications of WTs in different areas, are increasingly gaining importance in power quality researches. Optimal connection of WTs to the distribution networks is important in order to keep harmonic distortions within the allowable limits. However, the increase in the installation of these sources in the distribution networks leads to violations of the harmonic limits specified in IEEE 519 standards. In this manner, the optimal penetration levels of WTs should be determined taking into account the harmonic parameters. In this study, the probabilistic optimization approach is proposed to achieve optimal WT penetration levels by considering load uncertainty and wind speed variability in active distribution systems considering harmonic distortions. This approach is important for proper network planning. The cumulative distribution functions for the power quality parameters at node 18 , which is the most vulnerable bus to harmonic currents, are given in Figure 8 for different confidence levels. As presented in Figure 8, the VTHD at bus 18 has been observed above 5\% with probabilities of $0.0026,0.0034$, and 0.0001 , while the VIHD5 at bus 18 has been determined beyond the limit of $3 \%$ with probabilities of $0.3,0.2$, and 0.0993 for $0.7,0.8$, and 0.9 confidence levels respectively. Moreover, the VRMS has violated its corresponding limits with $0.0093,0.0288$, and 0.0123 probabilities for the confidence intervals of $0.7,0.8$, and 0.9 . Within the scope of this study, probabilistic harmonic analysis studies considering distorted active distribution networks to which WTs and nonlinear loads are connected have been carried out, and optimal penetration levels of these sources have been evaluated by considering the harmonic power quality issues with respect to wind speed. In WT connected distribution networks, changes in wind speed may cause violations of the harmonic parameters. In this manner, the probabilistic nature of harmonic parameters based on wind speed variations should be included in the constrained optimization problem while determining the optimal WT penetration levels by considering harmonic distortions in distribution networks. The probabilistic dependence of harmonic power quality parameters on changes in wind speed is an important research subject in the WT-integrated active distribution networks. In this study, the VTHD, VIHDh, and VRMS in distribution system vary stochastically depending on the wind speed due to the changes in operating points. Therefore, the effects of WTs on the harmonic parameters in the distribution networks are taken into consideration for variable wind speed in the harmonic analysis performed in the study. 
(a)

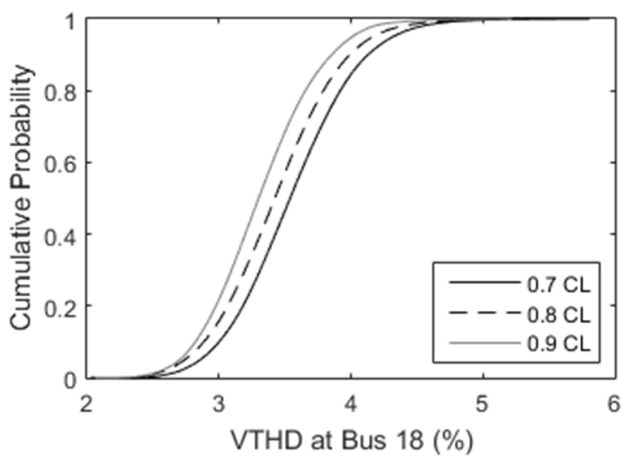

(b)

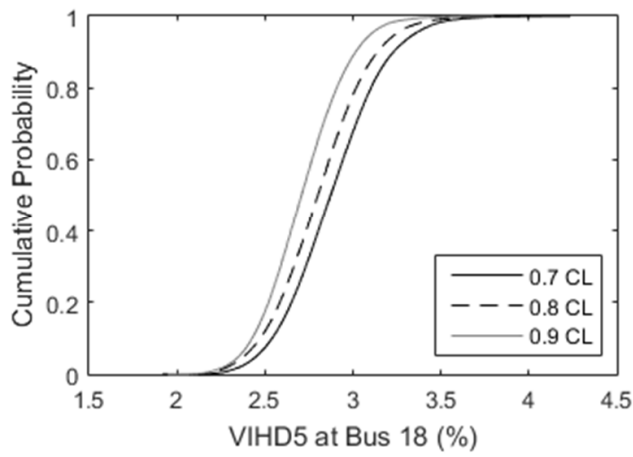

(c)

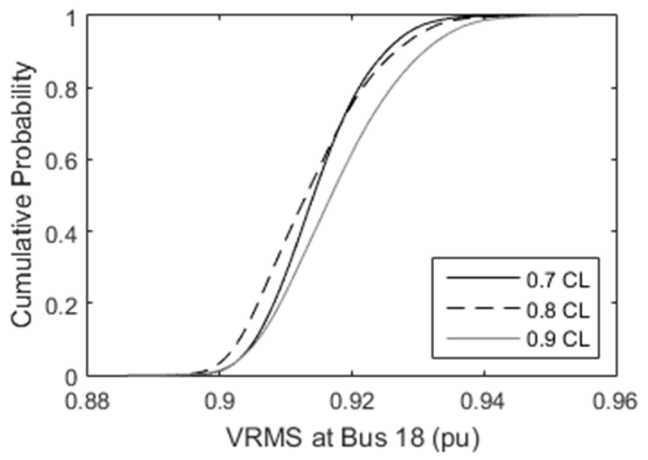

Fig. 8. The cumulative distribution functions for (a) VTHD, (b) VIHD5, (c) VRMS at bus 18 .

The assessment of harmonic power quality issues due to the WT presence for various wind speed states is a prominent topic considered in applications. In addition, determining the optimal output powers and penetration levels of the WTs integrated in the distribution networks is a promising problem in terms of keeping the VRMS, VIHDh, and VTHD values obtained from harmonic power flow analyses within the desired limits for the continuity and reliability of power quality. In this manner, the load profile of the distribution network, the variability of the capacity of the WTs depending on wind speed, and the harmonic constraints specified in the standards increase the importance of the problem of determining the optimal interconnections of the WTs in the distribution networks.

\section{CONCLUSIONS}

In this study, the uncertainties of wind speed and load profile in the distribution network are considered while dealing with the optimal WT penetration level problem in the stochastic optimization framework. The dependency of harmonic power quality problems on wind speed is handled in the WTs installed grid by the proposed method. By implementing GA along with MCS, the optimal WT penetration levels are determined by utilizing chance constraint programming. Harmonic power quality indices such as VTHD, VIHDh, and VRMS, which are considered while writing the chance constraints, are calculated by harmonic power flow analysis. Based on the results, the optimal capacities of WTs ensure that the violations in the chance constraints of the harmonic issues comply with the corresponding limits for each confidence level. Since the optimization process is more conservative in higher confidence levels, the optimal WT penetration levels decrease with the increase of confidence levels. The load uncertainty of distribution networks and the variability of wind speed on WTs encourage network operators to probabilistic planning studies. The main reason is that the stochastic optimization problem, which needs to be solved, has a complex structure due to the changes in load profile and wind speed. The dependence of the harmonic power quality of the grid on the operating points and wind speed adds a new dimension to this probabilistic optimization problem. Based on these points, the stochastic planning of optimal WT penetration levels in distribution networks brings the work done in the literature to a new level in terms of maintaining the power quality by minimizing the harmonic distortions. Therefore, the probabilistic planning on optimal WT penetration levels within the scope of this study has an important place in the relevant literature.

\section{ACKNOWLEDGMENT}

The author would like to thank Assoc. Prof. Dr. Engin Karatepe for his valuable and constructive comments to this paper.

\section{REFERENCES}

[1] G. Mustafa, M. H. Baloch, S. H. Qazi, S. Tahir, N. Khan, and B. A. Mirjat, "Experimental investigation and control of a hybrid (PV-Wind) energy power system," Engineering, Technology \& Applied Science Research, vol. 11, no. 1, pp. 6781-6786, Feb. 2021, https://doi.org/ 10.48084/etasr.3964.

[2] U. Vargas, G. C. Lazaroiu, and A. Ramirez, "Experimental validation of a hybrid TD/FEHD model of a wind turbine generator for harmonic transient analysis," Electric Power Systems Research, vol. 163, pp. 4958, Oct. 2018, https://doi.org/10.1016/j.epsr.2018.05.025.

[3] Z. Yuan, W. Wang, and X. Fan, "Back propagation neural network clustering architecture for stability enhancement and harmonic suppression in wind turbines for smart cities," Computers \& Electrical Engineering, vol. 74, pp. 105-116, Mar. 2019, https://doi.org/10.1016/ j.compeleceng.2019.01.006.

[4] R. Boopathi, R. Jayanthi, and M. M. T. Ansari, "Maximum power point tracking-based hybrid pulse width modulation for harmonic reduction in wind energy conversion systems," Computers \& Electrical Engineering, vol. 86, Sept. 2020, Art. no. 106711, https://doi.org/10.1016/ j.compeleceng.2020.106711.

[5] M. H. J. Bollen and K. Yang, "Harmonic aspects of wind power integration," Journal of Modern Power Systems and Clean Energy, vol. 1, no. 1, pp. 14-21, Jun. 2013, https://doi.org/10.1007/s40565-013-00017.

[6] T. Thiringer, T. Petru, and C. Liljegren, "Power quality impact of a sea located hybrid wind park," IEEE Transactions on Energy Conversion, vol. 16, no. 2, pp. 123-127, Jun. 2001, https://doi.org/10.1109/ 60.921462 . 
[7] J. H. Teng, R. C. Leou, C. Y. Chang, and S. Y. Chan, "Harmonic current predictors for wind turbines," Energies, vol. 6, no. 3, pp. 1314-1328, Mar. 2013, https://doi.org/10.3390/en6031314.

[8] S. T. Tentzerakis and S. A. Papathanassiou, "An investigation of the harmonic emissions of wind turbines," IEEE Transactions on Energy Conversion, vol. 22, no. 1, pp. 150-158, Feb. 2007, https://doi.org/ 10.1109/TEC.2006.889607.

[9] L. Sainz, J. J. Mesas, R. Teodorescu, and P. Rodriguez, "Deterministic and stochastic study of wind farm harmonic currents," IEEE Transactions on Energy Conversion, vol. 25, no. 4, pp. 1071-1080, Jun. 2010, https://doi.org/10.1109/TEC.2010.2045379.

[10] S. Liang, Q. Hu, and W. J. Lee, "A survey of harmonic emissions of a commercially operated wind farm," IEEE Transactions on Industry Applications, vol. 48, no. 3, pp. 1115-1123, Apr. 2012, https://doi.org/ 10.1109/TIA.2012.2190702.

[11] V. R. Pandi, H. H. Zeineldin, and W. Xiao, "Determining optimal location and size of distributed generation resources considering harmonic and protection coordination limits," IEEE Transactions on Power Systems, vol. 28, no. 2, pp. 1245-1254, Aug. 2012, https://doi.org/ 10.1109/TPWRS.2012.2209687.

[12] V. Hengsritawat, and T. Tayjasanant, "Impacts of load models and power factor control on optimal sizing of photovoltaic distributed generators in a distribution system," IEEJ Transactions on Electrical and Electronic Engineering, vol. 7, no. 6, pp. 567-573, Sept. 2012, https://doi.org/10.1002/tee.21774.

[13] Y. Zhao, H. Deng, J. Li, and D. Xia, "Optimal planning of harmonic filters on distribution systems by chance constrained programming," Electric Power Systems Research, vol. 68, no. 2, pp. 149-156, Feb. 2004, https://doi.org/10.1016/S0378-7796(03)00156-1.

[14] M. R. Jannesar, A. Sedighi, M. Savaghebi, A. Anvari-Moghaddam, and J. M. Guerrero, "Optimal probabilistic planning of passive harmonic filters in distribution networks with high penetration of photovoltaic generation," International Journal of Electrical Power \& Energy Systems, vol. 110, pp. 332-348, Sept. 2019, https://doi.org/10.1016/ j.ijepes.2019.03.025.

[15] A. Ulinuha, M. A. S. Masoum, and S. M. Islam, "Harmonic power flow calculations for a large power system with multiple nonlinear loads using decoupled approach," in 2007 Australasian Universities Power Engineering Conference, Perth, WA, Australia, Dec. 2007, pp. 1-6, https://doi.org/10.1109/AUPEC.2007.4548132.

[16] V. Kumar and S. K. Dhull, "Genetic Algorithm based optimization of uniform circular array," Engineering, Technology \& Applied Science Research, vol. 10, no. 6, pp. 6403-6409, Dec. 2020, https://doi.org/ 10.48084/etasr.3792.

[17] J. Zhu, Optimization of power system operation, 2nd ed. Hoboken, NJ, USA: John Wiley \& Sons, 2015.

[18] D.C. Montgomery and G.C. Runger, Applied Statistics and Probability for Engineers, 5th ed. Hoboken, NJ, USA: John Wiley \& Sons, 2010.

[19] "Recommended Practices and Requirements for Harmonic Control in Electric Power Systems,"IEEE Standard 519-2014, 2014.

[20] U. H. Ramadhani, M. Shepero, J. Munkhammar, J. Widen, and N. Etherden, "Review of probabilistic load flow approaches for power distribution systems with photovoltaic generation and electric vehicle charging," International Journal of Electrical Power \& Energy Systems, vol. 120, Sept. 2020, Art. no. 106003, https://doi.org/10.1016/ j.ijepes.2020.106003.

[21] Y. M. Atwa, E. F. El-Saadany, M. M. A. Salama, and R. Seethapathy, "Optimal renewable resources mix for distribution system energy loss minimization," IEEE Transactions on Power Systems, vol. 25, no. 1, pp. 360-370, Oct. 2009, https://doi.org/10.1109/TPWRS.2009.2030276.

[22] M. Milovanovic, J. Radosavljevic, and B. Perovic, "A backward/forward sweep power flow method for harmonic polluted radial distribution systems with distributed generation units," International Transactions on Electrical Energy Systems, vol. 30, no. 5, Dec. 2019, Art. no. e12310, https://doi.org/10.1002/2050-7038.12310.

[23] G. Papaefthymiou, P. H. Schavemaker, L. van der Sluis, W. L. Kling, D. Kurowicka, and R. M. Cooke, "Integration of stochastic generation in power systems," International Journal of Electrical Power \& Energy
Systems, vol. 28, no. 9, pp. 655-667, Nov. 2006, https://doi.org/10.1016/ j.ijepes.2006.03.004.

[24] A. Sehgal, H. M. La, S. J. Louis, and H. Nguyen, "Deep reinforcement learning using genetic algorithm for parameter optimization," in 2019 Third IEEE International Conference on Robotic Computing, Naples, Italy, Feb. 2019, pp. 596-601, https://doi.org/10.1109/IRC.2019.00121.

[25] A. Faza, "A probabilistic model for estimating the effects of photovoltaic sources on the power systems reliability," Reliability Engineering \& System Safety, vol. 171, pp. 67-77, Mar. 2018, https://doi.org/10.1016/ j.ress.2017.11.008

\section{AUTHORS PROFILE}

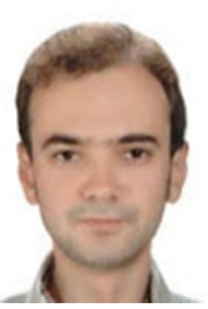

Ibrahim Cagri Barutcu received his B.S. (in 2011) degree from the Department of Electrical - Electronics Engineering in Eskisehir Osmangazi University, Eskisehir, Turkey, his M.S. (in 2014) and Ph.D. (in 2020) degrees from the Electrical - Electronics Engineering Department in Ege University, Izmir, Turkey. He is currently an Assisstant Professor in the Department of Electricity and Energy at Hakkari University. His research interests are harmonic power quality in active distribution networks, renewable energy resources, distributed generation planning and optimization methods. 